\section{Cahiers de Narratologie}

Analyse et théorie narratives

$33 \mid 2018$

L'Art du roman chez Umberto Eco

\title{
La construction de l'espace chez Umberto Eco
}

\section{Kamal Hayani El Mechkouri}

\section{OpenEdition}

\section{Journals}

Electronic version

URL: http://journals.openedition.org/narratologie/8071

DOI: 10.4000/narratologie.8071

ISSN: 1765-307X

\section{Publisher}

LIRCES

\section{Electronic reference}

Kamal Hayani El Mechkouri, « La construction de l'espace chez Umberto Eco », Cahiers de Narratologie [Online], 33 | 2018, Online since 23 July 2018, connection on 10 December 2020. URL : http:// journals.openedition.org/narratologie/8071; DOI : https://doi.org/10.4000/narratologie.8071

This text was automatically generated on 10 December 2020.

Article L.111-1 du Code de la propriété intellectuelle. 


\title{
La construction de l'espace chez Umberto Eco
}

\author{
Kamal Hayani El Mechkouri
}

1 L'étude de l'espace et son rôle dans la construction du sens n'a retenu l'attention des chercheurs qu'après la Seconde Guerre Mondiale. Les premières tentatives étaient pour le moins timides et limitées, l'espace étant jusque-là considéré comme un contenant, un enrichissement subordonné à l'intrigue qui primait sur tout autre composante de l'œuvre, comme l'affirme Jean Rousset :

« Si l'œuvre est dans sa forme, elle est complète et significative telle que l'artiste l'a composée, poème concret, roman achevé, et le reste ne devrait apporter que des enrichissements, des démentis, ou des moyens de fixer une éventuelle évolution $»^{1}$.

Grâce à La poétique de l'espace de Gaston Bachelard, publiée en 1961, la composante spatiale devient primordiale dans la construction du sens, en osmose avec les autres composantes intrinsèques et extrinsèques du texte. Dans le présent travail, nous nous proposons d'éclairer le processus de signification auquel participe le cadre spatial dans la lecture des œuvres d'Umberto Eco. Dans un premier temps, nous essayerons de repérer les espaces clos et les espaces ouverts exploités dans chaque roman, en vue de déterminer l'apport de chacun d'eux à la nature de la trame romanesque et aux personnages mis en contexte. Dans un second temps, nous insisterons sur les techniques d'Umberto Eco pour l'élaboration de ces espaces.

3 La devise du sémioticien italien est de voir le sens là où on serait tenté de ne voir que des faits. Ses écrits romanesques se présentent comme une mise en application de ses théories. Suivant les principes de la théorie de la coopération textuelle, Umberto Eco pousse son lecteur à collaborer dans la construction du sens, donc à adopter sa devise et à voir du sens dans tous les objets en récit. L'espace est l'un de ces objets que l'auteur cherche à faire parler, voire le plus important, car, selon ses propres dires :

«Le récit est d'abord et avant tout une affaire cosmologique. Pour raconter quelque chose, on commence par jouer le rôle d'une sorte de démiurge qui crée un monde, et ce monde doit être aussi précis que possible pour qu'on puisse s'y mouvoir en totale confiance $»^{2}$. 
4 Pour qu'il y ait confiance, les personnages doivent évoluer dans des espaces riches et surdéterminés. De ce fait, l'espace clos et l'espace ouvert exploités dans les textes d'Eco sont dictés par la nature de la trame de chaque roman et par la psychologie des personnages mis en récit.

\section{L'espace clos}

$5 \quad$ Le Nom de la rose est un roman policier dont l'action est située au Moyen Âge. L'objectif des enquêteurs, Guillaume de Baskerville et Bernard Gui, est d'assurer la sérénité du lieu en mettant fin aux troubles. Les personnages sont des moines s'évertuant à purifier leur âme des péchés et à la sauver du diable. De telles caractéristiques de la trame et de l'état des personnages dictent un espace clos et isolé du reste du monde. C'est la raison pour laquelle l'architecture du monastère bénédictin où se déroule l'action est faite dans le dessein de l'isoler et de le protéger. C'est une « construction octogonale qui, vue de loin, apparaissait comme une tétragone (figure absolument parfaite qui exprime la solidité et le caractère inexpugnable de la Cité de Dieu), dont les côtés méridionaux se dressaient sur le plateau de l'abbaye, tandis qu'au septentrion ils paraissaient s'élever des pentes même du mont d'où ils s'innervaient à pic $^{3}$ ».

6 Dans cet espace clos et bien protégé, les moines vivent aisément et en autarcie grâce aux dîmes, et ne donnent aux pauvres habitants que l'immondice qu'ils jettent du haut des murailles. L'abbaye est présentée comme une forteresse inaccessible, située sur une monumentale falaise et siégée par une herse et des tours qui surplombent des précipices. Si les murailles séparent l'intérieur de l'extérieur du monastère, il faut toutefois signaler que l'intérieur même est subdivisé en plusieurs autres espaces clos, sous une forme labyrinthique qui constitue une mise en abyme :

«Après la porte (qui était l'unique passage dans les murs d'enceinte) s'ouvrait une allée bordée d'arbres qui menait à l'église abbatiale. A gauche de l'allée s'étendaient une vaste zone de potagers [...], autour des deux édifices des balnea et de l'hôpital et herboristerie, qui épousaient la courbe de la muraille. Sur le fond, à gauche de l'église se dressait l'édifice, séparé de l'église par une esplanade recouverte de tombes. [...] à droite de l'église, s'étendaient certaines constructions qui se trouvaient derrière elle et autour du cloître : à coup sûr le dortoir, la résidence de l'Abbé et l'hôtellerie vers où nous dirigions nos pas et que nous atteignîmes en traversant un beau jardin. Sur le côté droit, au-delà d'une vaste esplanade, le long de la muraille méridionale et continuant à l'orient derrière l'église, une série de bâtiments agricoles, étables, moulins, pressoirs, greniers et caves, et ce qui me sembla être le bâtiment des novices $»^{4}$.

7 Il s'agit donc d'un espace clos contenant d'autres espaces clos tel une structure en gigogne : les cellules des moines, le réfectoire, les cuisines, la forge, l'herboristerie, le grenier, la salle de conseil et le scriptorium sont des espaces accessibles, et la crypte menant aux souterrains est un lieu secret, alors que la bibliothèque, « la plus grande bibliothèque de la chrétienté $»^{5}$, est un espace inaccessible et même interdit dont la violation constitue une véritable aventure, elle est au cœur d'un croisement labyrinthique de galeries, de chambres d'échos piégées ou en trompe-l'œil, comme une citadelle dressée pour défendre l'abbaye tout en restant loin de la vie sociale et politique du pays.

8 La fermeture de l'abbaye et la structure labyrinthique des lieux reflètent en quelque sorte la difficulté de l'enquête due à la résistance des signes. Ce n'est qu'en recourant à 
la conjecture et à l'abduction que Guillaume arrive à faire dire à ces mêmes signes ce qu'ils refusent de donner. En outre, la structure du roman est faite de façon à faire entrer le lecteur dans cet espace et ne le laisser sortir qu'à la fin du récit coïncidant avec le dénouement de l'affaire. Dès le début, le lecteur se trouve confronté à des pages difficilement compréhensibles, car, Umberto Eco affirme que «si quelqu'un voulait entrer dans l'abbaye et y vivre sept jours, il devait en accepter le rythme. S'il n'y arrive pas, il ne réussirait jamais à lire le livre dans son entier ${ }^{6}$. Ces premières pages font figure donc d'obstacles dont le dépassement permet au lecteur d'accéder à l'abbaye et d'y vivre pendant sept jours, elles sont le reflet de son architecture et ont « une fonction pénitentielle et initiatique $»^{7}$. Nous, lecteurs, nous y restons enfermés et nous ne nous libérons qu'avec la sortie de Guillaume et de son novice après la fin apocalyptique, l'incendie de toute l'abbaye.

La dénonciation de l'ésotérisme est le thème principal du deuxième roman Le Pendule de Foucault, ce qui explique la place importante accordée aux espaces clos. Dans ce roman, l'action commence et se termine dans des lieux fermés. A partir d'une maison d'édition appelée Garamond, Casaubon, Belbo et Diotallevi inventent un Plan pour la domination du monde à partir d'un prétendu document que les Templiers auraient rédigé avant la suppression de leur ordre. Ces derniers croient au plan imaginaire des trois amis et enlèvent et tuent Belbo en le pendant au pendule de Foucault, le même sort était réservé à Diotallevi. Casaubon, qui avait assisté à l'exécution de son ami, se réfugie dans la maison de campagne de Belbo située dans les Langhe. Cet espace clos et sécurisé donne le calme et la sérénité de l'esprit, et permet à Casaubon de raconter l'histoire à tête reposée, après avoir compris la vérité et la gravité de l'invention. Sur le plan narratif, cet endroit sert de repère double, il est le point d'arrivée de l'histoire et en même temps le point de départ de la narration rétrospective que le personnage principal entreprend.

10 Le troisième roman L'Ile du jour d'avant met en évidence une action qui se déroule dans un espace très vaste. Le personnage principal, Roberto de la Grive, part avec son père et une troupe de paysans pour défendre la forteresse en 1630. Rescapé, il quitte la Grive pour Aix-en-Provence puis pour Paris, d'où il part, bien malgré lui, dans une mission d'espionnage pour le compte de Mazarin en s'embarquant sur l'Amaryllis, un navire hollandais qui cache des activités secrètes. Le bateau fait naufrage et Roberto se trouve emprisonné sur la Daphné dans les îles Fidji en Mélanésie. Les deux principaux lieux fermés exploités dans le roman, la forteresse de Casal et la Daphné, constituent les nœuds de la trame romanesque.

11 La forteresse assiégée de Casal constitue le nœud de la vie de Roberto de la Grive et du roman. Le nœud d'accumulation, de surcharge d'expériences, de rencontres, enlacement, entrelacement de fils qui se croisent dans l'histoire de Roberto et influencent ses pensées. Le deuxième locus est celui de la Daphné où Roberto s'est retrouvé prisonnier loin de la côte. C'est le point final de ses pérégrinations, ou encore une pause lui permettant de découvrir, d'abord, les soubassements du bateau et le secret des activités cachées à bord, puis, son propre intérieur et ses histoires d'amour pour Lilia et celle de haine pour son alter ego ou son frère présumé Ferrante.

La Daphné représente la nature, les Terre incognita et l'avenir, puisque tout ce qui préoccupe Roberto sur le bateau est de pouvoir arriver à la côte, sa vie et son avenir dépendant de la réalisation de cet objectif. Tandis que l'autre locus, Casal, incarne la guerre et le passé du héros. Toutefois, les deux espaces se rencontrent comme étant des 
lieux d'apprentissage et de formation pour le personnage principal. La forteresse lui a permis de découvrir le monde extérieur et ses normes, alors que la Daphné lui a offert le calme et la disjonction du monde extérieur pour concentrer ses efforts sur son nouveau petit monde et sur son propre intérieur. Mais tout cela est destiné à meubler en quelque sorte le roman, car le plus important est la trouvaille spatiale qui consiste à faire naufrage en face d'une île qui appartient à hier, c'est-à-dire à ce qui n'est plus, la mort.

Dans Baudolino, roman exclusivement réservé à la puissance du mensonge, l'espace subit la même contamination; le royaume du prêtre Jean en est la parfaite illustration. Il semble qu'un lieu fermé représente le repos dont ont besoin les personnages pour réfléchir et donner leurs propres interprétations liées à leurs états d'âme. Le lieu fermé, est, semble-t-il également, une pause pour l'écrivain lui-même pour relancer l'action et lui donner d'autres issues. Le cas de la chambre close dans Baudolino est très illustratif à cet égard.

La chambre en question a été offerte à l'empereur Frédéric Barberousse par l'arménien Arzdrouni qui était chargé de guider les troupes impériales vers l'Arménie. La clôture de la chambre est renforcée par son emplacement dans le château-forteresse de Dadjig en Cilicie, c'est un château-forteresse aussi inhospitalier que le château des Carpates de Jules Verne, il est perché, perdu et presque oublié de son propriétaire, hors du temps et de l'espace humain. Umberto Eco, pour sa part, s'attarde sur la description de l'atmosphère qui règne autour et dans la chambre : l'environnement et les positions de l'armée de la garde, les étranges appareils qui se trouvent dans la forteresse et les expériences du mage Arzdrouni. C'est un espace qui fait peur par ce qu'il contient de machines inconnues aux allures étranges et par le chemin escarpé qui mène à l'entrée.

La mort de l'empereur dans cette chambre représente pour Umberto Eco un motif fictionnel qu'il exploite pour ouvrir le récit sur l'imaginaire médiéval. Cet incident constitue un point stratégique; il met fin à la première partie du roman, considérée proprement de façon historique ou, du moins, réaliste, et ouvre le récit sur un point important de l'imaginaire médiéval, en laissant Baudolino et ses amis à poursuivre leur quête du royaume du Prêtre Jean.

Dans La Mystérieuse Flamme de la reine Loana, Umberto Eco exploite la symbolique des lieux de mémoire à travers un lieu singulier, celui de l'enfance, incarnée ici par Solara. Yambo qui a perdu la mémoire et qui dispose de tout le roman pour la retrouver, se réveille à l'hôpital et commence à nous relater les différentes vicissitudes qui constitueront l'intrigue du roman. Le récit se lance donc à partir d'un espace clos, qui est la chambre de l'hôpital, comme une enceinte génitrice de création, une matrice de procréation et de naissance. Amnésique qu'il est, le personnage décrit tout ce qu'il voit avec de minutieux détails, il se compare à Adam qui découvre son jardin de l'Eden.

Le décor aseptisé de la chambre d'hôpital moderne et impersonnelle reflète l'état psychique du personnage et sa recherche du "Moi ", ses actes manqués sont dus à des incursions de son inconscient. Ils sont en quelque sorte le parler vrai du moi le plus profond, constituant aussi un point de départ pour la recherche matérielle dans les différents recoins, mais aussi un point de départ pour les épanchements et les états d'âme. D'une mémoire blanche et sans souvenirs précis, sauf quelques lueurs indistinctes et perdues au milieu du brouillard inconscient, il entame la recherche de son Moi intime, et à mesure que ses découvertes "matérielles » avancent, il ressent 
aussi de mystérieuses flammes l'innerver et partir à l'élaboration d'une trame aux multiples méandres.

Sous les conseils du médecin et de sa femme, il passe quelques jours dans la maison de sa petite enfance, appelée Solara. Sur le chemin, il nous décrit le paysage qui défile derrière les vitres de la voiture telle une séquence cinématographique, et, dès son arrivée, il présente la maison et les différentes pièces-espaces dont elle est constituée : les chambres, le grenier, la chapelle, etc. Gaston Bachelard nous dit que la maison « est une des plus grandes puissances d'intégration pour les pensées, les souvenirs et les rêves de l'homme ${ }^{8}$ ». C'est pour récupérer ses pensées, ses souvenirs et ses rêves que Yambo scrute tous les objets dans les différents recoins de la maison. Les gravures colorées des images d'Epinal, les gravures allemandes qui recouvrent les parois où tous les meubles de la vaste cuisine et la table de la salle à manger provoquent chez lui de vagues réminiscences. Dans ce sens, Gaston Bachelard affirme que "grâce à la maison, un grand nombre de nos souvenirs sont logés et si la maison se complique un peu, si elle a cave et grenier, des coins et des couloirs, nos souvenirs ont des refuges de mieux en mieux caractérisés ${ }^{9} »$.

Espace hautement symbolique, le grenier représente un univers retiré et protégé, contenant à son tour d'autres espaces clos "minimes", des cartons, des boîtes, des coffres et des armoires. Ces espaces minimes lui donnent la sensation de renaitre et en constituent les terminaisons nerveuses et mnémoniques : «j'étais en train d'éprouver une satisfaction ancienne [...] me rappeler de confuses expériences précédentes ${ }^{10} \gg$ ditil. Quant à la chapelle, où Yambo aimait se réfugier et se cacher, elle est murée et interdite aux autres. Elle lui apporte une retraite intime et lui permet de se concentrer sur lui-même. La remontée au grenier et la découverte tardive de la chapelle constituent une sorte de progression dans la recherche. Les multiples réminiscences s'accentuent au fur et à mesure de son avancement, et l'arrivée dans la chapelle constitue l'ultime étape qui lui permet d'accéder à ce quelque chose qui résiste encore.

Dans Le cimetière de Prague, on relève deux aspects ayant favorisé l'emploi du lieu fermé dans les précédents romans et qu'Umberto Eco réexploite dans ce dernier roman, à savoir : les trous de mémoire repérés chez Yambo de La mystérieuse Flamme de la reine Loana et le thème du complot et de la conspiration, traité dans Le Pendule de Foucault. L'action commence à l'appartement de Simon Simonini, et comme Yambo, celui-ci a des trous de mémoire et se sent poursuivi par les autres. Dans l'appartement, il a l'impression qu'il n'est pas seul quand il trouve, suspendu à un cintre, dans son placard, des vêtements de prêtre. Il a le sentiment que quelqu'un d'autre, en l'occurrence l'abbé Dalla Piccola, vient s'immiscer chez lui pendant son absence. Ce double avait permis à Simonini d'affiner ses recherches en corrigeant ou en proposant des synthèses d'événements qu'il raconte dans son journal intime. Après l'avoir tué, signe de guérison de l'état schizophrénique, Simonini avoue que : «Dalla Piccola avait servi pour fouiner dans les milieux satanistes et occultistes ${ }^{11}$. C'est grâce à cet alter-ego qu'il a pu rencontrer Taxil et Boullan et intégrer le milieu des jésuites et des satanistes et décrire leurs espaces servant d'officine à leurs rituels secrets.

21 En outre, les missions d'espionnage, dont le personnage principal est chargé, le conduisent vers des lieux fermés où sont cachés ou détenus les conspirationnistes. Dans ce sens, la prison pour Simon Simonini n'a pas un rôle correctionnel, mais celui d'un lieu fermé de planification et de préparation de l'étape suivante, et ce, à travers la collecte des informations dans une mission d'espionnage. Il y rentre comme étant un 
réfugié mazzinien ayant des rapports avec des républicains français afin de faire parler Maurice Joly, détenu lui aussi dans la même prison pour avoir publié Dialogue aux enfers entre Machiavel et Montesquieu, et aussi pour rencontrer Gaviali, qui a eu quelque chose à voir avec l'attentat d'Orsini.

De façon générale, nous constatons que l'espace clos est exploité dans tous les romans d'Umberto Eco dans un sens strictement sémiotique. Comme les signes, les lieux dictent aux personnages des actions, des fois-mêmes ces mêmes lieux sont des actants à part entière, qui jouent un rôle de premier ordre dans les transformations narratives. Ainsi, en s'articulant, l'espace finit par inscrire en lui une série d'actions de ceux qui y vivent. Les personnages des romans d'Eco évoluent dans des lieux qu'ils subissent parfois, resémantisent d'autre fois ou adaptent à leurs propres exigences. Dans tous ces cas, les lieux ne sont plus un simple abri, destiné à contenir, mais un élément actif et déterminant dans l'économie globale du roman.

\section{L'espace ouvert}

Après cette mise au point sur les différentes manifestations de l'espace clos dans les romans d'Umberto Eco, nous focalisons notre attention, à présent, sur la part de l'espace ouvert et son rapport à la fabula de chaque récit. Dans le premier roman, Le Nom de la Rose, l'action se déroule pendant les sept jours dans une abbaye fermée et l'espace ouvert représente l'extérieur qui est quasiment absent. Il incarne le milieu du mal, des ignorants, des pauvres, des frénétiques, etc. La même remarque est à faire de La Mystérieuse Flamme de la reine Loana, on y assiste à une absence d'espace ouvert, hormis ce que le personnage principal décrit lors de son voyage de l'hôpital à Solara ou les évocations de la nature environnante. Dans sa route vers Solar, Yambo nous livre une description qui ressemble à bien des égards à une séquence cinématographique et l'œil du personnage se substitue à celui de la caméra. Il décrit le paysage mais en étant derrière les vitres de la voiture, écran certes, mais paroi entre un dehors et un dedans. L'insertion de cette brèche d'espace ouvert est un clin d'œil au brouillard et à la mystérieuse flamme que le personnage amnésique ressent de temps à autre, c'est une brève sortie d'une clôture de l'espace et de la mémoire.

Dans les autres romans, les personnages principaux se déplacent beaucoup, et ce, en parfaite osmose avec les transformations narratives ou les séries d'actions et de réactions qui sont déterminantes dans l'avancement de l'intrigue. Ce voyage acquiert tantôt une fonction informative, tantôt une fonction cathartique. Dans Le Pendule de Foucault, l'espace ouvert est le monde entier dont la domination reste le premier objectif. Cette ouverture totale est dictée par les impératifs du thème du complot. Plus le cadre est élargi, plus le complot a de l'impact : le complot juif, le complot américain, le complot des rose-croix, etc. La recherche de documentation, pour inventer le Plan, mène les personnages à plusieurs endroits. Plusieurs villes et pays sont parcourus, du Milan des manifestations politiques post-soixante-huitardes au Paris du Conservatoire des Arts et Métiers et de la Tour Eiffel, du Brésil des rituels afro-brésiliens aux Langhe des souvenirs de jeunesse de Belbo, en passant par Londres, magique et occulte, et par Prague, ésotérique et kabbalistique.

Les différents lieux visités jouent un rôle certes formatif pour le héros, il y fait des expériences qui marquent profondément son parcours intellectuel. Au Brésil, il rencontre le marquis Aglié, qui l'a subjugué par son érudition et ses riches 
connaissances sur l'ésotérisme et l'occultisme. A Milan, il a constaté que les idées et les valeurs sont changées, ce qui l'a poussé davantage vers le côté irrationnel. Au Piémont, il assiste, avec ses amis et Aglié, à un spectacle hallucinant et surnaturel qui lui a fait perdre la lumière de la raison. Et c'est à Paris, dans la nuit du 23 au 24 juin, au Conservatoire des Arts et Métiers, qu'il assiste au rendez-vous entre Belbo et ses ravisseurs.

En dépit de ce voyage formatif et initiatique de Casaubon dans Le Pendule de Foucault, l'action se déroule souvent dans des lieux fermés qu'exige une trame faisant de l'ésotérisme et du complot un de ses principaux thèmes. C'est pourquoi Umberto Eco a opté pour un espace plus ouvert dans L'T̂le du jour d'avant : "Après deux livres décrivant un lieu clos, un monastère et ce musée (les Arts et Métiers à Paris), je voulais respirer le grand air, aller dans la nature $»^{12}$.

Dans ce roman, Eco adopte également la technique rétrospective dans la narration.

Roberto de la Grive, une fois naufragé sur la Daphné, commence à relater à rebours ce qu'il a vécu avant, c'est ce que nous dit l'auteur lui-même : «la Daphné se transformait en Théâtre de la Mémoire, tel qu'on le concevait à son époque, où chaque morceau lui rappelait un épisode ancien ou récent de son histoire $»^{13}$. C'est donc, un voyage dans la mémoire du personnage principal, une forme d'introspection profonde. Autrement dit, une occasion pour le personnage schizophrène d'analyser sa personnalité et de mettre fin à ses soucis à l'égard de son frère présumé Ferrante, c'est pour cette raison qu'on peut qualifier ce voyage de cathartique aussi bien qu'initiatique.

28 A l'encontre de la beauté du paysage évoquée dans L'Ile du jour d'avant, l'espace ouvert dans Baudolino est un espace de débris et de troubles, reflétant l'état de l'Europe au XIIème siècle et incitant au déplacement, à la fuite et à la recherche d'un lieu meilleur. Cette ruine est également le reflet de l'état psychique du personnage éponyme, le menteur qui croit en ses mensonges et tombe victime de ce qu'il crée et de ce qu'il croit être réel. Ce mélange du vrai et du faux dépasse les faits relatés et atteint les repères géographiques qui, à mesure de l'avancement du récit, deviennent de plus en plus flous. Ce que le romancier tente de dire à propos de la superposition de lieux appartenant à notre monde actuel avec d'autres lieux appartenant au monde virtuel de personnage comme Baudolino, c'est que les deux types d'espace, quand bien même opposés, ont un seul et unique dénominateur commun : ce sont des signes intériorisés par l'homme qui le poussent à agir.

Baudolino voyage beaucoup avec l'empereur, puis poursuit seul ses pérégrinations. Il part étudier à Paris, puis se dirige vers l'Orient pour rejoindre un lieu inventé de toute pièce, le royaume du Prêtre Jean. La trame romanesque se base sur l'Histoire et sur un personnage témoin qui est le fils adoptif de Frédéric Barberousse, donc il va de soi que l'espace ouvert de tout l'empire germanique sera évoqué.

30 La description de l'espace lors du voyage vers l'Orient nous fait voir un univers tout à fait imaginaire, un univers où les repères géographiques sont brouillés et imprécis. En suivant les instructions de la carte de Cosmas, Baudolino et ses amis traversent Les plaines brûlées de Yambut, les marécages de Cataderche, les villes de Salibut, Caramaria, Salopatana, le fleuve Boubouctor, dont les pierres de la grève noircissent la peau de celui qui les touche, et le fleuve légendaire de pierre et de sable, Sambatyon. Les noms de ces territoires brouillent à dessein les repérages géographiques.

31 Cet embrouillement de l'espace reflète l'imprécision de la carte de Cosmas qui leur sert de guide, et fait écho à l'espace géographique du Moyen Âge qui ne se préoccupe pas de 
localisations ou de distances précises, mais qui a des valeurs symboliques, selon l'expression d'Henry Corbin ${ }^{14}$. Ici Eco exploite toutes ces connaissances sur les lieux légendaires et imaginaires pour inspirer ses personnages et nourrir l'intrigue. Sur ces valeurs symboliques, Youri Lotman nous dit que «se mouvoir dans l'espace géographique signifiait se déplacer le long de l'échelle verticale des valeurs religieuses et morales $»^{15}$. De ce fait, nous pouvons dire que le voyage terrestre dans Baudolino et ses multiples contraintes et périls renvoient au voyage que doit entreprendre l'homme à la recherche de la connaissance, de la conscience et de la foi.

L'espace ouvert évoqué dans Le Cimetière de Prague est un espace vaste s'étendant sur plusieurs pays. Simon Simonini est un espion multicarte, il pérégrine entre la France, l'Italie et l'Allemagne. A l'encontre de Baudolino, lors de ses multiples voyages, Simonini ne donne pas des descriptions détaillées des lieux parcourus. La raison est que ce roman peut être qualifié de roman à thèse, dont la thématique principale est Les protocoles des Sages de Sion. L'objectif d'Umberto Eco est d'affirmer la fausseté d'un document accusant les juifs d'avoir un plan pour conquérir le monde et mettre fin au catholicisme en Europe. Pour atteindre cet objectif, peu importe l'espace, l'auteur opte pour un personnage faussaire, dont la mission est d'affirmer l'existence du complot juif par le biais de mensonges et de falsifications.

\section{La conception de l'espace chez Eco}

Est-il possible après cette série d'exemples de cerner la conception de l'espace chez Eco. Une citation de l'auteur à ce sujet pourra nous aider à le faire :

"Le récit est d'abord et avant tout une affaire cosmologique. Pour raconter quelque chose, on commence par jouer le rôle d'une sorte de démiurge qui crée un monde, et ce monde doit être aussi précis que possible pour qu'on puisse s'y mouvoir en totale confiance $»^{16}$.

L'auteur fait mouvoir ses personnages grâce à la maîtrise des détails de cet univers. C'est pour cette raison qu'il investit beaucoup de temps dans l'élaboration du cadre spatial d'un roman avant même de commencer sa rédaction.

Le roman voit le jour après une longue période de recherche et d'enquête. Le principe d'Eco le romancier est qu'« il faut construire le monde, les mots viennent ensuite, presque tout seuls. Rem tene, verba sequentur $\aleph^{17}$, c'est pour cette raison qu'il passe son temps, pendant ses années de gestation littéraire, «à visiter des lieux et à dessiner des cartes; à noter les plans de bâtiments, voire d'un navire comme dans L'Ile du jour d'avant $»^{18}$. Cette déclaration explique, en grande partie, l'exactitude des dimensions des lieux à la réalité et l'effet du réalisme donné à certains lieux imaginaires. Et comme l'indique l'auteur, cette démarche peut être apparentée à celle de Luchino Visconti dans ses films. Quand le script indiquait que deux personnages parlaient d'une boite de bijoux, il tenait à ce que la boite, même si on ne l'ouvrait jamais, fût remplie de vrais bijoux. Sinon, les acteurs auraient joué avec moins de conviction.

Dans Le Nom de la rose, l'auteur met au début le plan de l'abbaye. D'abord pour aider le lecteur à bien visualiser le déplacement des personnages dans un espace labyrinthique difficilement accessible, puis pour mentionner dès le début que cet espace joue le rôle le plus important, sans le saisir on risque de perdre le fil conducteur des actions importantes. Bien que beaucoup de détails aient été empruntés à d'autres édifices réels, l'auteur affirme que cette abbaye ne correspond à aucune autre dans la réalité, il s'est 
inspiré d'un manuscrit sans titre d'Athanase Kircher qu'il avait trouvé dans une boutique de livres anciens à Buenos Aires ${ }^{19}$.

Cette maîtrise de l'espace a poussé le cinéaste Marco Ferreri à dire à Umberto Eco : "votre livre semble conçu expressément pour qu'on en tire un scénario, car les dialogues ont exactement la bonne longueur $»^{20}$. Cette longueur correspond totalement à la distance entre les différents édifices du monastère. Pour créer un tel synchronisme, Eco avoue qu'il avait dessiné des centaines de labyrinthes et de plans d'abbayes, de sorte qu'il savait combien de temps il faudrait à deux personnages pour se rendre d'un lieu à un autre tout en conversant ${ }^{21}$.

Les lieux du Pendule de Foucault sont réels, Eco a passé pas mal de temps à les visiter et les parcourir, de sorte qu'il maîtrise les dédales de chaque espace évoqué ainsi que tout ce qui les entoure. Pour le Conservatoire des Arts et Métiers à Paris, il avoue avoir passé "des soirées entières jusqu'à l'heure de la fermeture "22 à arpenter ses couloirs. La plupart des événements de l'œuvre se passent entre le Conservatoire, la place des Vosges et la tour Eiffel. Eco nous confie la stratégie adoptée : «j'ai passé plusieurs nuits à parcourir la ville entre deux et trois heures du matin, murmurant dans un dictaphone pour prendre note de tout ce que je voyais ainsi ne pas me tromper dans les noms de rues et les croisements $»^{23}$.

39 L'étendue espace de L'Ile du jour d'avant n'a pas empêché l'auteur de s'y rendre avant la rédaction de son roman. Les îles de Fidji et la richesse des mers du sud sont décrites de façon précise et conforme à la réalité parce que l'auteur les a visitées pendant la préparation de son œuvre " pour observer les couleurs de la mer et du ciel à différentes heures du jour et les teintes des poissons et des coraux $»^{24}$. Concernant la Daphné, l'espace clos de l'ouvrage, l'auteur reconnaît avoir consacré « deux ou trois ans à l'étude des dessins et des modèles des navires de l'époque, pour connaitre les dimensions d'une cabine ou d'une coursive et savoir comment une personne pouvait s'y déplacer $\aleph^{25}$. Lorsqu'il parle de sa connaissance des dimensions du navire, il signifie plutôt que ces espaces sont calculés au millimètre comme s'il allait les fabriquer.

Quant aux espaces exploités dans Baudolino, Umberto Eco a fait de sa propre région un cadre spatial pour la première moitié du roman. La géographie de l'Italie en général et de sa petite ville natale en particulier constituent autant de lieux parcourus par Baudolino et l'armée impériale. Dans ce cadre, l'auteur italien dit : « Baudolino était une bonne occasion de revenir à mon Moyen Âge bien-aimé, à mes racines personnelles $»^{26}$.

41 En outre, le choix de Constantinople découle de la fascination qu'éprouve l'auteur pour cette ville qu'il n'avait jamais visitée. Pour expliquer ce choix, il dit :

«Pour avoir une raison de m'y rendre, j'avais besoin de raconter une histoire sur cette ville et sur la civilisation byzantine. Je fis donc le voyage de Constantinople. J'explorai la surface de la ville et les couches en dessous, et trouvai l'image fondatrice de mon récit : celle de Constantinople saccagée par les croisés en 1204 » 27.

De surcroît, le voyage vers l'Orient se base sur la carte de Cosmas, et c'est la même carte sur laquelle Umberto Eco s'est appuyé pour tracer les frontières et les contours des différentes contrées. Les données géographiques ne sont pas précises parce qu'elles sont le reflet de la géographie du Moyen Âge qui ne n'accordait pas une grande importance aux détails.

43 La Mystérieuse Flamme de la reine Loana est le roman le plus autobiographique. L'auteur puise certains événements dans ses souvenirs d'enfance. L'évocation de la maison de 
campagne de Solara est une allusion à la maison de son propre grand père ; il maîtrise à merveille son architecture ainsi que les différents espaces clos cités. Presque la même méthode a été adoptée dans le roman suivant, Le cimetière de Prague. Puisque la plupart des régions visitées par Simonini sont familières à l'écrivain ; les villes italiennes, Paris, etc. En outre, nous constatons que, dans les deux derniers romans, l'auteur ne fait pas de la description détaillée des lieux une priorité, et ce, pour mettre en relief les thématiques principales des œuvres, en l'occurrence : la mémoire, les Protocoles des sages de Sion et l'Histoire.

\section{Conclusion}

Les lieux chez Eco sont d'abord et avant toute chose une catégorie du récit et du sens au sens plein. Leur variété et leur diversité obéit au souci du romancier, qui est d'offrir un monde plein, il y va donc du bon fonctionnement de l'effet de la fable. En même temps, ces lieux demeurent étroitement liés aux thèmes chers à Eco, le secret, le complot, le mensonge et la mémoire. Cependant, en fonction de leur statut de catégorie de récit et de créateurs d'espace d'écriture, ils sont envisagés de façon rigoureusement sémiotique, c'est-à-dire comme des signes qui abritent les personnages mais qui finissent en même temps par les habiter, voire les hanter. Personnages et lieux s'éduquent et s'influencent mutuellement, tout comme les signes.

\section{BIBLIOGRAPHY}

Albert-Birot, Pierre. 1945. Les amusements naturels. Paris. Denoël.

Bachelard, Gaston. 1957. La poétique de l'espace. Paris. Les Presses universitaires de France.

Eco, Umberto. 1982. Le Nom de la rose. Paris. Grasset \& Fasquelle.

Eco, Umberto. 1990. Le Pendule de Foucault. Paris. Grasset \& Fasquelle.

Eco, Umberto. 1996. L'île du jour d'avant. Paris. Grasset \& Fasquelle.

Eco, Umberto. 2002. Baudolino. Paris. Grasset \& Fasquelle.

Eco, Umberto. 2005. La Mystérieuse Flamme de la reine Loana. Paris. Grasset \& Fasquelle.

Eco, Umberto. 2011. Le Cimetière de Prague. Paris. Grasset \& Fasquelle.

Eco, Umberto. 2013. Confessions d'un jeune romancier. Paris. Grasset \& Fasquelle.

Eco, Umberto. 1983. Apostille au Nom de la rose. Paris, Le livre de Poche.

Lotman, Youri. 1999. La sémiosphère. Trad. Anka Lodenko, Coll. « Nouveaux Actes Sémiotiques ». Limoges. Presses Universitaires de Limoges.

Nedjat, Hamid. 2005. Des mille et une nuits à la Geographie imaginalis persane. In Imaginaires des quatre points cardinaux. Paris. Imago. 
Rousset, Jean. 1962. Forme et signification. Essais sur les structures littéraires de Corneille à Claudel.

Paris. José Corti.

\section{NOTES}

1. Jean Rousset, Forme et signification. Essais sur les structures littéraires de Corneille à Claudel, Paris, José Corti, 1962, p. 20

2. Umberto Eco, Confessions d'un jeune romancier, Paris, Grasset et Fasquelle, 2013, p. 22

3. Umberto Eco, Le Nom de la rose, Paris, Grasset et Fasquelle, 1982, p. 33

4. Ibid. p. 39

5. Ibid. p. 496

6. Umberto Eco, Apostille au Nom de la rose, Paris, Le Livre de Poche, 1983, p. 49

7. Ibid.

8. Gaston Bachelard, La poétique de l'espace, Paris, PUF, 1957, p. 34

9. Ibid. p. 36

10. Umberto Eco, La Mystérieuse Flamme de la reine Loana, Paris, Grasset et Fasquelle, p. 117

11. Umberto Eco, Le Cimetière de Prague, Paris, Grasset et Fasquelle,, 2011, p. 498

12. Umberto Eco, Lire, $\mathrm{n}^{\circ} 243$, mars 1996, p. 41

13. Umberto Eco, L'Ile du jour d'avant, Paris, Grasset et Fasquelle, 1996, p. 109

14. H. Nedjat, Des mille et une nuits à la Geographia imaginalis persane, in Imaginaires des quatre points cardinaux, p. 45

15. Y. Lotman, La sémiosphère, Limoges, Presses Universitaires de Limoges, 1999, trad. Anka Lodenko, coll. « Nouveaux Actes Sémiotiques », p. 87.

16. Confessions d'un jeune romancier, op. cit., p. 22

17. Apostille au Nom de la rose, Op. Cit., p. 28

18. Confessions d'un jeune romancier, Op. Cit., p. 19

19. Ibid, p. 80

20. Ibid., p. 21

21. Ibid.

22. Ibid., p. 20

23. Ibid.

24. Ibid.

25. Ibid.

26. Ibid., p. 30

27. Confessions d'un jeune romancier, op. cit., p. 31

\section{ABSTRACTS}

The purpose of this study is to describe and analyze systematically the various types of places used by Eco in his novels and the design of the space that emerges. The article focuses on two fundamental types of space carried by Eco in almost all of his novels : namely closed spaces and open and unlimited space. These two types of space are selected because they create spaces of writing closely related to a poetics of the secret not to say of the plot. In this novelistic treatment 
of space, Eco mobilizes many semiotic reminiscences on the premises as restrictive but at the same time inspiring forms of character behavior.

L'objectif de cette étude est de décrire et d'analyser systématiquement les différents types de lieux mis en récit par Umberto Eco et la conception de l'espace qui s'en dégage. L'article fait le point sur deux types fondamentaux d'espace exploités par Eco dans presque tous ses romans : à savoir les espaces fermés et les espaces ouverts et illimités. Ces deux types sont choisis car ils créent des espaces d'écriture étroitement liés à une poétique du secret pour ne pas dire de l'intrigue. Dans ce traitement romanesque de l'espace, Eco mobilise une réflexion sémiotique sur les lieux comme des formes restrictives mais en même temps inspirantes du comportement des personnages. Il est question donc d'un paramètre que le sémioticien et romancier italien cherche à faire converser et dialoguer puisqu'il participe avec les autres données intrinsèques et extrinsèques du texte pour créer le sens et les sensations.

INDEX

Mots-clés: Eco, espace, lieux, poétique du secret, sémiotique de l'espace

Keywords: space, places, Secret, plot, semiotics of space, Eco

\section{AUTHOR}

KAMAL HAYANI EL MECHKOURI

Ecole doctorale, Université de Fès 